Original Article (short paper)

\title{
Rate of force development to evaluate the neuromuscular fatigue and recovery after an intermittent isometric handgrip task with different blood flow restriction conditions
}

\author{
Mikhail Santos Cerqueira ${ }^{1}$, Rafael Pereira ${ }^{2} \oplus$, Gabriel Nunes de Mesquita ${ }^{1}$, \\ Taciano Rocha ${ }^{3}$, Alberto Galvão de Moura Filho ${ }^{1}$ (1) \\ ${ }^{1}$ Universidade Federal de Pernambuco,UFPE, Department of Physical Therapy, Recife, PE, Brazil; \\ ${ }^{2}$ Universidade Estadual do Sudoeste da Bahia, UESB, Research Group in Neuromuscular \\ Physiology, Department of Biological Sciences, Jequié, BA, Brazil; ${ }^{3}$ Universidade Federal do \\ Rio Grade do Norte, UFRN, Department of Physical Therapy, Natal, RN, Brazil
}

\begin{abstract}
Aim: To investigate the neuromuscular fatigue and recovery after an intermittent isometric handgrip exercise (IIHE) executed until failure with different blood flow restriction (BFR) conditions (free flow, partial and total vascular restriction). Methods: Thirteen healthy men carried out an IIHE at $45 \%$ of maximum voluntary isometric force (MVIF) until failure with total restriction (TR), partial restriction (PR) or free flow (FF). The rate of force development (RFD) was extracted from the MIVF over the time intervals of $0-30,0-50,0-100$, and $0-200 \mathrm{~ms}$ and normalized by MVIF [relative RFD (RFDr)]. Results: The RFDr decreased significantly $(p<0.01)$ after the IIHE in all BFR conditions and time intervals studied, remaining lower for five minutes. The medians of the RFDr in FF condition were significantly lower $(p=0.01)$ at $30 \mathrm{~ms}\left(1.56 \% \mathrm{MVIF} \cdot \mathrm{s}^{-1}\right)$ and $50 \mathrm{~ms}\left(1.70 \% \mathrm{MVIF} \cdot \mathrm{s}^{-1}\right)$ when compared to TR at $30 \mathrm{~ms}\left(2.34 \% \mathrm{MVIF} \cdot \mathrm{s}^{-1}\right)$ and 50ms (2.63\%MVIF $\left.\cdot \mathrm{s}^{-1}\right)$ in minute 1 post failure. Conclusions: These results show that, regardless of the blood flow restriction level, there is no RFD recovery five minutes after an exhaustive IIHE. When the task was executed with FF, the reduction of the RFD was greater when compared with the TR condition.
\end{abstract}

Keywords: vascular occlusion; fatigue; task failure; muscle strength.

\section{Introduction}

Blood flow during a muscle effort is continuously controlled to ensure nutrient and oxygen support, as well as the metabolites withdrawal from the exercised muscles, allowing an optimal muscle performance and recovery ${ }^{1}$. Paradoxically, blood flow restriction (BFR) during muscle efforts has been used in training routines with promising gains in muscle strength and mass ${ }^{2,3}$. Interestingly, BFR induces many metabolic/hormonal/neural acute responses associated with positive muscle adaptations ${ }^{4}$, but obligate an earlier task failure (TF), especially when higher BFR pressures are applied during the exercise ${ }^{5}$. Usually, partial BFR instead of total BFR has been used with similar gains in strength and mass $^{6}$. Despite this, the knowledge regarding the mechanisms involved in the TF induced by BFR during muscle efforts has not been widely explored.

$\mathrm{TF}$ is defined as the inability to keep performing a requested task. Physiologically, it has specific mechanisms, involving central and peripheral factors, which depends on task characteristics ${ }^{7,8}$. Despite the obvious mutual interaction between central and peripheral mechanisms ${ }^{8}$, it is clear that in different situations (type of contraction, intensity, duration, blood flow condition, ambient temperature $)^{9,10}$ there is a predominance of one of these mechanisms leading to the TF. The mechanisms of TF may be partially understood by events that occur after failure ${ }^{7,11,12}$. Nevertheless, some neuromuscular parameters have allowed the investigation of neural and muscular factors contribution to muscle force development in fatigue or TF contexts, as the rate of force development (RFD) ${ }^{13}$.

The RFD, which is derived from the force- or torque-time curves recorded during maximal voluntary contractions ${ }^{14}$, have been increasingly used to characterize the capacity for rapid muscle force production in populations including athletes and frail elderly patients ${ }^{15}$. Among reasons for this interest are the facts that (1) it is sensitive to detect acute and chronic changes in neuromuscular function ${ }^{15}$, (2) estimate the degree of fatigue and recovery after acute exhausting exercise ${ }^{16}$ and (3) be potentially governed by different physiological features ${ }^{15,17}$, such as neural and muscular (viz., peripheral) mechanisms. Despite the peak force, defined as the maximum force achieved during a maximal muscle effort, independently of the time to achieve it, be a widely used parameter to study muscle performance, there are some limitations with this parameter, when the aim is to investigate the mechanisms involved in the muscle force production capacity ${ }^{18}$, when compared to the RFD. Buckthorpe, Pain, Folland ${ }^{18}$ demonstrated that the fatigue exerts a more pronounced influence on RFD than on peak force production, being suggested that both, contractile and neural mechanisms were responsible. Thus, it was evidenced that RFD is more suitable than peak force to investigate neuromuscular and mechanical events involved in muscle performance decline after a fatigable task. 
Notably, recent studies have indicated that a single bout of low-load partial BFR exercise may acutely $(\leq 1 \mathrm{~h})$ exert a negative influence on maximal muscle strength ${ }^{5,19}$. Yet, it remains unknown how the application of different levels of BFR influences on recovery of RFD after the exercise. Given this, the present study aimed to investigate the neuromuscular fatigue and recovery after an intermittent isometric handgrip exercise (IIHE) executed until failure with different blood flow levels (free flow, partial and total vascular restriction). This might improve the knowledge regarding the predominance of neural and peripheral mechanisms involved in the TF induced by BFR.

\section{Methods}

\section{Subjects}

Thirteen men classified as physically active or very active according to the International Physical Activity Questionnaire (IPAQ) volunteered to participate in this study. All subjects denied the use of any medication, smoking or the diagnostic of the neurological, orthopedic or cardiovascular disease. Participants were instructed to avoid strenuous handgrip exercise, maintain the same level of physical activity and diet during the study period, as well as to avoid alcohol and caffeine consumption in the 24 hours antecedent to the experimental procedures. Local Ethics Committee approved all experimental procedures (CAAE: 36832814.9.0000.5208) in accordance with the Declaration of Helsinki, and the volunteers were informed about possible risks and signed a consent form before the study beginning. This study was prospectively registered on Clinicaltrials.gov (NCT02384161).

\section{Experimental procedures}

Volunteers were submitted to three sessions of intermittent isometric handgrip exercise (IIHE) sustained until failure with three different blood flow conditions: total restriction (TR), partial restriction (PR) and free blood flow (FF). The interval between two successive interventions ranged from $72 \mathrm{~h}$ (minimum interval) and 1 week (maximum interval). Thus, in this crossover study design, all volunteers carried out the same IIHE up to failure with all blood flow conditions (TR, PR, FF) in random order.

Through computer-generated random number tables (http://www.randomization.com/) the order of blood flow conditions was allocated for each subject by an investigator who was not involved in the recruitment, intervention or assessment of participants. Opaque sealed envelopes were used to conceal the allocation. To blind all procedures, the researcher responsible for maximum voluntary isometric force (MVIF) assessment and control of the IIHE (researcher 1) did not know volunteers allocation. The researchers responsible to determine (researcher 2) and apply/control the occlusion pressure (researcher 3) did not participate in the randomization process. The volunteers were not informed about the level of occlusion applied in each day and were instructed to avoid reporting the perception of pressure, keeping researcher 1 blinded regarding the used occlusion pressure.

Initially, blood pressure and anthropometric data were collected from volunteers. The following day, the total occlusion pressure (TOP) of the brachial artery was determined, and the volunteers were familiarized to the assessment of MVIF and the IIHE. Seventy-two hours after the familiarization session, volunteers began the IIHE sessions with different blood flow conditions. Thenceforth, each IIHE session was identical, except for the blood flow condition, as described following. The volunteers were positioned in dorsal decubitus, with shoulder abducted at $90^{\circ}$, elbow fully extended and forearm supinated for all TOP and MVIF evaluations and during the IIHE.

\section{Total occlusion pressure determination}

TOP is a measure that shows good reproducibility ${ }^{20}$ and was determined according to the previous studies ${ }^{5,20}$. Briefly, the brachial artery blood flow of a dominant arm was detected by an ultrasound model SonoAceR3 (Samsung Medison - South Korea), by Power Doppler Technique - with $12 \mathrm{MHz}$ linear transducers placed in the flexor crease of the elbow. Visual and auditory signals indicated the presence of pulse during the cuff inflation (Aneroid sphygmomanometer Premium, Duque de Caxias - RJ; width cuff $14 \mathrm{~cm}$ ). The TOP was determined as the lowest pressure necessary to occlude completely the blood flow, and the partial occlusion pressure was set as the value corresponding to $50 \%$ of TOP.

\section{Maximal voluntary isometric force and intermittent isometric handgrip exercise}

After standing for five minutes in a climatized room $\left(23^{\circ} \mathrm{C}\right)$, the subjects were submitted to MVIF assessments. In all sessions of IIHE, the MVIF of a dominant arm was assessed before (PRE) and at minutes 1, 3 and 5 after the TF (POST 1-POST 5) using a custom-made strain gauge-based force transducer (DM 100, Miotec, Porto Alegre, RS, Brazil). Recordings were sampled at $2000 \mathrm{~Hz}$ and subjects were carefully instructed to contract "as fast and forcefully as possible" after the command "go," sustained the contraction for $5 \mathrm{~s}$, when the command "stop" was given ${ }^{13}$.

In the PRE measures, each volunteer performed three attempts of MVIF with 1 minute of rest between contractions. The highest of the three attempts was used to calculate the force target to be used along the IIHE, which was set as $45 \%$ of MVIF. Five minutes after the third MVIF pre-exercise attempt, the volunteers started the IIHE, carrying out successive isometric handgrip contractions, each of one sustained for 10 seconds, followed by 5 -seconds of rest, repeated until failure. This procedure was done three days apart, each one with the TR, PR or FF condition. Volunteers were instructed to reach and maintain the target force ( $45 \%$ of MVIF) as brief and accurately as possible during the active phase, and relax (force $=0$ ) during the resting phase. The periods of action and resting were controlled by a metronome-timed beep sound. 
The active/rest cycles were repeated until TF, which was defined as the incapacity to reach and/or sustain the force greater than $30 \%$ of MVIF for 5 seconds or more in three consecutive active phases (figure 1). Along the task, the force data collected by a strain gauge- based force transducer (DM 100, Miotec, Porto
Alegre, RS, Brazil) was displayed by projection on the ceiling located approximately two meters away from the volunteer. To determine the time to failure during IIHE in TR, PR and FF conditions the number of repetitions were measured according to Cerqueira et $\mathrm{al}^{5}$.

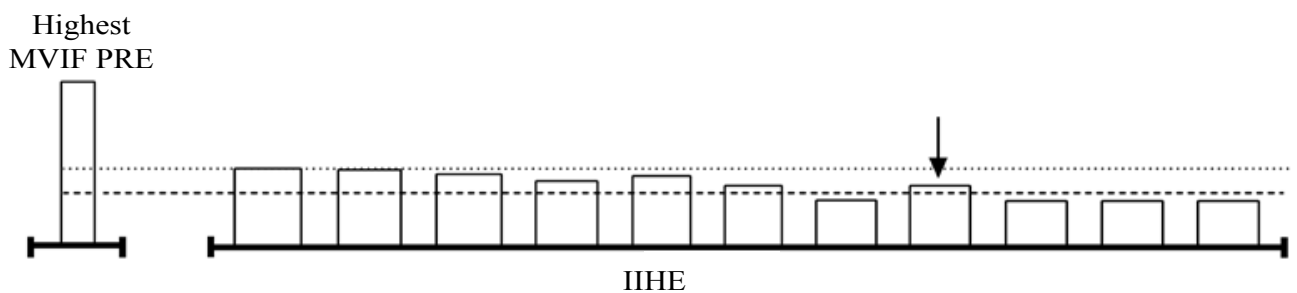

Figure 1. A timeline describing the experimental procedures. The superior line indicates the target force ( $45 \%$ of maximal voluntary isometric force [MVIF]). The inferior line indicates the cutoff force to consider task failure (i.e., the minimum force [30\% of MVIF]). The black arrow indicates the last valid contraction, since the inability to sustain the force above the cutoff ( $30 \%$ of MVIF) for three consecutive contractions was established as the criteria for task failure. IIHE: Intermittent isometric handgrip exercise.

In FF condition the cuff was inflated to a pressure sufficient only to adjust to the volunteer's arm $(\sim 10 \mathrm{mmHg})$, thus maintaining the forearm blood flow free. Meanwhile, at PR and TR conditions, the cuff was inflated with a pressure of $50 \%$ and $100 \%$ of the TOP brachial artery, respectively. The same cuff used to determine the TOP (positioned at the dominant arm, just below the axilla, near to the insertion of the deltoid muscle) was inflated immediately before and deflated immediately after the end of IIHE. During the MVIF assessments and during IIHE, volunteers received strong verbal stimuli from researcher 1. Single MVIF measurements were performed after the TF (i.e., at POST 1 - POST 5 assessments). All MVIF measures were performed with free blood flow.

\section{Rate of force development analysis}

For the handgrip RFD analysis, the strain gauge signal extracted from the MIVF curves was smoothed by a digital fourth-order, zero-lag Butterworth filter, with a cutoff frequency of $15 \mathrm{~Hz}$, as proposed by Aagaard, Simonsen, Andersen, Magnusson, Dyhre-Poulsen ${ }^{14}$, and used by Schettino et $\mathrm{al}^{21}$. The average slope of the force-time curve ( $\Delta$ force/ $\Delta$ time) over the time intervals of $0-30,0-50,0-100$, and $0-200 \mathrm{~ms}$ relative to the onset of contraction was calculated and is representative of $\mathrm{RFD}^{14}$. The onset of muscle contraction was defined as the time point at which the force curve exceeded the baseline by $2.5 \%$ of the difference between baseline force and the MVIF (i.e., maximum handgrip force) ${ }^{14,21}$. The RFD was also normalized (relative RFD - RFDr) by the MVIF (\%MVIF $\cdot \mathrm{s}^{-1}$, i.e., RFD at the peak force) and calculated at $30,50,100$ and $200 \mathrm{~ms}$ after the onset ${ }^{22,23}$. All analyses were conducted using specific routines developed in MATLAB 7.0.1 ${ }^{\circledR}$ (MathWorks Inc).

\section{Statistical analyses}

The Shapiro-Wilk test was used to determine whether the variables were normally distributed. Since the variables were not normally distributed, the data were presented as median and interquartile range $\left(25^{\text {th }}\right.$ and $75^{\text {th }}$ percentiles), and the Friedman's test followed by Wilcoxon's test with Bonferroni post hoc correction test when necessary, was used to examine the differences in RFDr from 0 to 200ms between moments (PRE, POST1, POST3, and POST5) and conditions (TR, PR, FF). A significance level of $p<0.05$ was used for all statistical procedures, and all statistical analyses were performed using SPSS 18.0 (IBM Corp., Chicago, IL,USA).

\section{Results}

The participant's characteristics are shown in Table 1. The baseline values of MVIF (in Newtons) were 445.33 \pm 75.30 ; $447.17 \pm 79.43$ and $448.08 \pm 74.72$ for TR, PR and FF conditions, respectively. Time to failure values (in seconds) was $150 \pm 68$; $390 \pm 210 ; 510 \pm 240$ for TR, PR and FF conditions, respectively.

Table 1. Characteristics of the participants $(n=13)$. Values are mean \pm standard deviation, minimum and maximum.

\begin{tabular}{lccc}
\hline Variable & Mean \pm SD & Minimum & Maximum \\
\hline Age $($ years $)$ & $21 \pm 1.7$ & 19 & 25 \\
Heigth $(\mathrm{cm})$ & $177 \pm 6$ & 170 & 187 \\
Body mass $(\mathrm{kg})$ & $78.58 \pm 9.5$ & 61.5 & 91.8 \\
BMI $\left(\mathrm{kg} / \mathrm{m}^{-2}\right)$ & $25.03 \pm 1.9$ & 21.3 & 28.5 \\
SBP $(\mathrm{mmHg})$ & $120.15 \pm 11.6$ & 100 & 140 \\
DBP $(\mathrm{mmHg})$ & $76.92 \pm 7.51$ & 70 & 90 \\
TRP $(\mathrm{mmHg})$ & $126.92 \pm 10.5$ & 110 & 145 \\
PRP $(\mathrm{mmHg})$ & $63.46 \pm 5.3$ & 55 & 72.5 \\
\hline
\end{tabular}

BMI, body mass index; SBP, systolic blood pressure; DBP, diastolic blood pressure; TRP, total restriction pressure and PRP, partial restriction pressure 
Table 2 presents the RFDr values in the time intervals of $0-30$, 0-50, 0-100, and 0-200ms from TR, PR and FF conditions measured previously and 1, 3 and 5 minutes after IIHE. In all BFR conditions and time intervals, the RFDr values decreased significantly after IIHE when compared with baseline, remaining lower up to 5 minutes post failure $(p<0.01)$.
Differences between BFR conditions were observed only 1 minute after failure and at the time intervals of $0-30,0-50 \mathrm{~ms}$. In the FF, condition the RFDr values were significantly lower at $30 \mathrm{~ms}$ when compared with TR $(p=0.01)$ and PR conditions $(p=0.004)$. At $50 \mathrm{~ms}$ the RFDr values in the FF condition were lower only when compared to TR $(p=0.014)$.

Table 2. The relative rate of force development (\%MVIF-s-1) obtained before (PRE) and post 1 (POST1), 3 (POST3), and 5 (POST5) minutes after task failure at TR, PR and FF conditions.

\begin{tabular}{|c|c|c|c|}
\hline & TR & PR & FF \\
\hline \multicolumn{4}{|c|}{ RFDr - PRE } \\
\hline $0-30 \mathrm{~ms}$ & $3.33(2.41-4.43)$ & $3.86(2.08-4.48)$ & $2.49(2.31-4.54)$ \\
\hline $0-50 \mathrm{~ms}$ & $4(2.84-5.31)$ & $4.54(2.47-5.36)$ & $3.01(2.81-5.24)$ \\
\hline $0-100 \mathrm{~ms}$ & $4.70(3.51-6.01)$ & $5.34(3.07-6.01)$ & $3.71(3.45-5.44)$ \\
\hline $0-200 \mathrm{~ms}$ & $3.72(3.33-4.05)$ & $3.69(2.89-4.28)$ & $3.35(2.97-3.92)$ \\
\hline \multicolumn{4}{|c|}{ RFDr - POST1 } \\
\hline $0-30 \mathrm{~ms}$ & $2.34(1.55-2.84)$ & $1.38(1.09-2.19)$ & $1.56^{\dagger}(0.63-1.72)$ \\
\hline $0-50 \mathrm{~ms}$ & $2.63(1.76-3.25)$ & $1.57(1.16-2.55)$ & $1.70^{\dagger \dagger}(0.61-2)$ \\
\hline $0-100 \mathrm{~ms}$ & $2.67(1.90-3.31)$ & $1.59(1.14-2.93)$ & $1.86(0.61-2.27)$ \\
\hline $0-200 \mathrm{~ms}$ & $1.88(1.32-2.41)$ & $1.29(0.83-2.74)$ & $1.60(0.78-1.92)$ \\
\hline \multicolumn{4}{|c|}{ RFDr - POST3 } \\
\hline $0-30 \mathrm{~ms}$ & $2.24(1.34-2.43)$ & $1.28(0.81-2.21)$ & $1.30(0.80-2.52)$ \\
\hline $0-50 \mathrm{~ms}$ & $2.6(1.72-2.83)$ & $1.48(0.91-2.58)$ & $1.51(0.88-2.85)$ \\
\hline $0-100 \mathrm{~ms}$ & $2.97(2.26-3.15)$ & $1.75(1.11-3.07)$ & $1.76(1.06-2.98)$ \\
\hline $0-200 \mathrm{~ms}$ & $2.34(1.95-2.70)$ & $1.50(1.10-2.66)$ & $1.69(1.08-2.50)$ \\
\hline \multicolumn{4}{|c|}{ RFDr - POST5 } \\
\hline $0-30 \mathrm{~ms}$ & $1.92(1.41-2.80)$ & $1.76(1.09-2.31)$ & $1.26(1.04-2.08)$ \\
\hline $0-50 \mathrm{~ms}$ & $2.24(1.65-3.26)$ & $2.10(1.19-2.64)$ & $1.49(1.15-2.53)$ \\
\hline $0-100 \mathrm{~ms}$ & $2.81(2.10-3.60)$ & $2.32(1.27-2.99)$ & $1.85(1.22-3.10)$ \\
\hline $0-200 \mathrm{~ms}$ & $2.48(2-3.02)$ & $1.77(0.99-2.43)$ & $1.95(1.30-2.68)$ \\
\hline
\end{tabular}

TR: total restriction; PR: partial restriction; FF: free flow; RFDr: relative rate of force development; ms: milliseconds. $(*)$ Significantly different from POST1, POST3, and POST5, independently of a blood flow condition; ( $\dagger$ ) significantly different from TR and PR conditions; ( $\dagger$ ) significantly different from TR condition. Values are the median with the $25^{\text {th }}$ and $75^{\text {th }}$ percentiles.

\section{Discussion}

The purpose of this study was to investigate the neuromuscular fatigue and recovery after an intermittent isometric handgrip exercise (IIHE) executed until failure with different blood flow levels. The major finding was that the RFDr declined significantly after the exhaustive protocol, remaining below to the baseline measure up to 5 minutes, independently of blood flow condition. Additionally, the FF condition exhibited significantly lower RFDr at 30 and $50 \mathrm{~ms}$, when compared with TO condition.

The literature about the RFD after BFR exercises are scarce, however, a recent study showed that three weeks of BFR training may impair the muscle contractile properties for at least five days after exercise interruption ${ }^{2}$. An acute study verified that the maximum torque was lower eight minutes after four sets of low-intensity (30\% 1RM) knee extension with partial BFR ( $60 \%$ of arterial occlusion pressure) or FF when compared with baseline measure ${ }^{24}$. However, in this study, the exercise was not performed until failure and RFD was not evaluated.

The ability to develop rapid muscle force production is influenced by many factors, such as the output from the central nervous system ${ }^{14,15}$, proportion of fast twitch fibers ${ }^{25}$ and passive mechanical properties of the muscle-tendon complex. ${ }^{26}$ Notwithstanding, it is proposed that RFD is influenced by 
different factors at early $(<100 \mathrm{~ms})$ and late phases $(>100 \mathrm{~ms})$ from the onset of muscle contraction, ${ }^{27}$ with consistent evidences that the early phase is directly influenced by neural drive and the late phase by muscle properties ${ }^{15,27}$.

We found a significant decline in early and late RFDr (30-200 $\mathrm{ms}$ ) along 5 minutes after the exercise protocol, independently of blood flow condition. The decline in the early phase of RFDr (i.e, $<100 \mathrm{~ms}$ ) along the five minutes after the IIHE may be justified by alterations in neural factors such as reduction of the motor unit discharge rate $e^{15,16}$. Although the applied protocol has no features to induce changes in muscle structure properties, the decrease in the late RFDr after TF may relate to impairment of cross-bridge kinetics ${ }^{13,28,29}$ imposed by the accumulation of metabolites, as well as the exercise-induced reactive oxygen/nitrogen species production in the muscle fiber ${ }^{30}$. In fact, our results from the FF condition corroborate the results from Buckthorpe, Pain, Folland $^{18}$, which demonstrated a significant decline in the RFDr after a fatigable task consisted of many cycles of action/rest. Owing to use of the RFD analysis, the authors could evidence that both, neural and contractile fatigue mechanisms contribute to the decline in muscle force performance, but with a greater influence of neural mechanisms, since the early phase of RFDr was greatly influenced. Additionally, Buckthorpe, Pain, Folland ${ }^{18}$ evidenced that RFD is more suitable than peak force to investigate mechanisms involved muscle performance decline after a fatigable task.

Regarding the comparisons between blood flow conditions, we found the greatest decline at the early phase of contraction (RFDr at 30 and $50 \mathrm{~ms}$ ) at the FF condition one minute after the exhaustive protocol. As expected, FF condition presented a greater time to achieve the task failure, which may be associated to a markedly greater neural inhibition, induced by afferent sensory nerve fibers (groups III and IV) ${ }^{31-33}$. This postulate is in line with previous founds, which indicated that the magnitude of force reduction after the TF is strongly influenced by the exercise duration $^{5}$. In fact, the slower force recovery after the TF observed in the task sustained for a long period (i.e., FF condition), may occur owing to deficiencies in the contractile apparatus, the membrane excitability, and excitation-contraction coupling ${ }^{34}$.

The short follow up after TF (i.e., 5 minutes) limited the identification of necessary time for a complete recovery of the capacity for rapid muscle force production after the failure at all blood flow conditions exercises. However, it is important to note that the short-term recovery, as applied to recovery between sets of an exercise (e.g., weight training or sprints), involve intervals equal or less than 5 minutes $^{35}$, and the use of ergogenic supplements aiming to improve the short-term recovery, as creatine supplementation, appears to convey no advantage for recovery durations greater than 6 minutes $^{36}$. Then, further studies could investigate this issue, reproducing our experimental design, which includes the short follow up after TF (i.e., 5 minutes), to extend the knowledge regarding the use of ergogenic supplements on a short-term recovery of muscle force parameters at different BFR conditions.

The use of a handgrip task can be considered a limitation of our study since most studies on BFR exercise involve other muscle groups (such as knee extensors or elbow flexors).
However, we considered the use of handgrip task adequate for the present study, since neuromuscular fatigue was induced by a task that involves the precision (i.e., maintenance of a target force with a maximal precision). Another limitation of our study is that only men were included in the sample, which limits the extrapolation to women, especially because there are sex-dependent characteristics in the recovery after fatiguing tasks ${ }^{12}$. However, our results pave the way for future studies designed to achieve these two pending aspects, which were out of the scope of the present study.

\section{Conclusions}

Regardless of a blood flow condition, RFD declined immediately after an exhaustive intermittent isometric handgrip task and does not return to the pre-exercise levels for the following five minutes. Additionally, the free flow condition was associated to a greater decline in the early phase (i.e., in the first $100 \mathrm{~ms}$ after the contraction onset) of RFD when compared to the task executed with total blood flow restriction, indicating a markedly greater neural inhibition, induced by afferent sensory nerve fibers (groups III and IV), since free flow condition was also associated to a greater time to achieve the task failure. These findings improve the knowledge regarding the neuromuscular response to exercises with different blood flow restriction conditions, paving the way to future studies investigating the neuromuscular adaptations to the exercise training with blood flow restriction.

\section{References}

1. Joyner MJ, Casey DP. Regulation of increased blood flow (hyperemia) to muscles during exercise: a hierarchy of competing physiological needs. Physiol Rev. 2015;95(2):549-601.

2. Nielsen JL, Frandsen U, Prokhorova T, Bech RD, Nygaard T, Suetta $\mathrm{C}$, et al. Delayed effect of blood flow-restricted resistance training on rapid force capacity. Med Sci Sports Exerc. 2017;49(6):1157-1167.

3. Lixandrão ME, Ugrinowitsch C, Berton R, Vechin FC, Conceição MS, Damas F, et al. Magnitude of Muscle Strength and Mass Adaptations Between High-Load Resistance Training Versus Low-Load Resistance Training Associated with Blood-Flow Restriction: A Systematic Review and Meta-Analysis. Sports Med. 2018;48(2):361-378.

4. Pearson SJ, Hussain SR. A Review on the Mechanisms of BloodFlow Restriction Resistance Training-Induced Muscle Hypertrophy. Sports Med. 2014;45(2):187-200.

5. Cerqueira MS, Pereira R, Rocha T, Mesquita G, Lima CROP, Raposo MCF, et al. Time to failure and neuromuscular response to intermittent isometric exercise at different levels of vascular occlusion: a randomized crossover study. Int J App Exerc Physiol. 2017;6(1):55-70.

6. Lixandrão ME, Ugrinowitsch C, Laurentino G, Libardi CA, Aihara AY, Cardoso FN, et al. Effects of exercise intensity and occlusion pressure after 12 weeks of resistance training with blood-flow restriction. Eur J App Physiol. 2015;115(12):2471-2480. 
7. Hunter SK, Duchateau J, Enoka RM. Muscle fatigue and the mechanisms of task failure. Exerc Sport Sci Rev. 2004;32(2):44-49.

8. Barry BK, Enoka RM. The neurobiology of muscle fatigue: 15 years later. Integr Comp Biol. 2007;47(4):465-473.

9. Moritani T, Sherman WM, Shibata M, Matsumoto T, Shinohara M. Oxygen availability and motor unit activity in humans. Eur J Appl Physiol Occup. Physiol. 1992;64(6):552-556.

10. Enoka RM, editor: Neuromechanics of human movement. 4th Edition. Champaign, Human Kinetics. 2013.

11. Neyroud D, Maffiuletti NA, Kayser B, Place N. Mechanisms of fatigue and task failure induced by sustained submaximal contractions. Med Sci Sports Exerc. 2012;44(7):1243-1251.

12. Senefeld J, Pereira HM, Elliott N, Yoon T, Hunter SK. Sex Differences in Mechanisms of Recovery after Isometric and Dynamic Fatiguing Tasks. Med Sci Sports Exerc. 2018;50(5):1070-1083.

13. Peñailillo L, Blazevich A, Numazawa $H$, Nosaka K. Rate of force development as a measure of muscle damage. Scand J Med Sci Sport. 2015;25(3):417-427.

14. Aagaard P, Simonsen EB, Andersen JL, Magnusson P, DyhrePoulsen P. Increased rate of force development and neural drive of human skeletal muscle following resistance training. J Appl Physiol. 2002;93(4):1318-1326.

15. Maffiuletti NA, Aagaard P, Blazevich AJ, Folland J, Tillin N, Duchateau J. Rate of force development: physiological and methodological considerations. Eur J App Physiol. 2016;116(6):1091-116.

16. Rodríguez-Rosell D, Pareja-Blanco F, Aagaard P, González-Badillo JJ. Physiological and methodological aspects of rate of force development assessment in human skeletal muscle. Clin Physiol Funct Imaging. 2017; doi: 10.1111/cpf.12495.

17. Andersen LL, Aagaard P. Influence of maximal muscle strength and intrinsic muscle contractile properties on contractile rate of force development. Eur J App Physiol. 2006;96(1):46-52.

18. Buckthorpe M, Pain MT, Folland JP. Central fatigue contributes to the greater reductions in explosive than maximal strength with high-intensity fatigue. Exp Physiol. 2014;99(7):964-973.

19. Cook SB, Murphy BG, Labarbera KE. Neuromuscular function after a bout of low-load blood flow-restricted exercise. Med Sci Sports Exerc. 2013;45(1):67-74.

20. Bezerra de Morais AT, Santos Cerqueira M, Moreira Sales R, Rocha T, Galvão de Moura Filho A. Upper limbs total occlusion pressure assessment: Doppler ultrasound reproducibility and determination of predictive variables. Clin Physiol Funct Imaging. 2017;37(4):437-441.

21. Schettino L, Luz CPN, De Oliveira LEG, de Assunção PL, Silva Coqueiro R, Fernandes MH, et al. Comparison of explosive force between young and elderly women: Evidence of an earlier decline from explosive force. Age. 2014;36(2):893-898.

22. de Oliveira FBD, Rizatto GF, Denadai BS. Are early and late rate of force development differently influenced by fast-velocity resistance training? Clin Physiol Funct Imaging. 2013;33(4):282-287.

23. Oliveira FBD, Oliveira ASC, Rizatto GF, Denadai BS. Resistance training for explosive and maximal strength: Effects on early and late rate of force development. Journal Sports Sci Med. 2013;12(3):402-408.

24. Husmann F, Mittlmeier T, Bruhn S, Zschorlich V, Behrens M. Impact of Blood Flow Restriction Exercise on Muscle Fatigue Development and Recovery. Med Sci Sports Exerc. 2018;50(3):436-446.

25. Harridge SD, Bottinelli R, Canepari M, Pellegrino MA, Reggiani $\mathrm{C}$, Esbjörnsson $\mathrm{M}$, et al. Whole-muscle and single-fibre contractile properties and myosin heavy chain isoforms in humans. Pflugers Archiv:Eur J Physiol. 1996;432(5):913-920.

26. Bojsen-Møller J, Magnusson SP, Rasmussen LR, Kjaer M, Aagaard P. Muscle performance during maximal isometric and dynamic contractions is influenced by the stiffness of the tendinous structures. J Appl Physiol. 2005;99(3):986-994.

27. Oliveira AS, Corvino RB, Caputo F, Aagaard P, Denadai BS. Effects of fast-velocity eccentric resistance training on early and late rate of force development. Eur J Sport Sci. 2016;16(2):199-205.

28. Farup J, Rahbek SK, Bjerre J, de Paoli F, Vissing K. Associated decrements in rate of force development and neural drive after maximal eccentric exercise. Scand J Med Sci Sport. 2016;26(5):498-506.

29. Edman KAP, Josephson RK. Determinants of force rise time during isometric contraction of frog muscle fibres. J Physiol. 2007;580(Pt.3):1007-1019.

30. Cheng AJ, Yamada T, Rassier DE, Andersson DC, Westerblad H, Lanner JT. Reactive oxygen/nitrogen species and contractile function in skeletal muscle during fatigue and recovery. J Physiol. 2016;594(18):5149-5160.

31. Gandevia SC. Spinal and supraspinal factors in human muscle fatigue. Physiol Rev. 2001;81(4):1725-1789.

32. Kaufman MP, Rybicki KJ. Discharge properties of group III and IV muscle afferents: their responses to mechanical and metabolic stimuli. Circ Res. 1987;61(4 Pt 2):I60-5.

33. Garland SJ. Role of small diameter afferents in reflex inhibition during human muscle fatigue. J Physiol. 1991;435:547-558.

34. Pitcher JB, Miles TS. Influence of muscle blood flow on fatigue during intermittent human hand-grip exercise and recovery. Clin Exp Pharmacol Physiol. 1997;24(7):471-476.

35. Bishop PA, Jones E, Woods AK. Recovery from training: a brief review: brief review. J Strength Cond Res. 2008;22(3):1015-1024.

36. Seiler S, Hetlelid KJ. The impact of rest duration on work intensity and RPE during interval training. Med Sci Sports Exerc. 2005;37(9):1601-1607.

\section{Acknowledgements}

This research was supported by grant: FACEPE APQ 0821-4.08 / 08, CNPq and CAPES PROCAD / NF 791/09. Mikhail Santos Cerqueira and Gabriel Nunes de Mesquita would like to thank Coordination for the Improvement of High Level (CAPES, Brazil), for the scholarship concession.

\section{Corresponding author}

Mikhail Santos Cerqueira

Departamento de Fisioterapia, Universidade Federal de Pernambuco, UFPE, Brasil.

Address: Jorn. Aníbal Fernandes av. s/n Cidade Universitária, Recife. $50740-560$

Email: mikalsantosc@hotmail.com

Manuscript received on June 25, 2018

Manuscript accepted on October 19, 2018

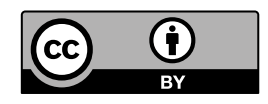

Motriz. The Journal of Physical Education. UNESP. Rio Claro, SP, Brazil - eISSN: 1980-6574 - under a license Creative Commons - Version 4.0 